\title{
A Novel Uncoded SER/BER Estimation Method
}

\author{
Mahesh Patel and A. Annamalai \\ Department of Electrical and Computer Engineering, Prairie View A \& M University, \\ TX 77446, United States of America
}

\begin{abstract}
Due to the rapidly increasing data speed requirement, it has become essential to smartly utilize the available frequency spectrum. In wireless communications systems, channel quality parameters are often used to enable resource allocation techniques that improve system capacity and user quality. The uncoded bit or symbol error rate (SER) is specified as an important parameter in the second and third generation partnership project (3GPP). Nonetheless, techniques to estimate the uncoded SER are usually not much published. This paper introduces a novel uncoded bit error rate (BER) estimation method using the accurate-bits sequence of the new channel codes over the AWGN channel. Here, we have used the new channel codes as a forward error correction coding scheme for our communication system. This paper also presents the simulation results to demonstrate and compare the estimation accuracy of the proposed method over the AWGN channel.
\end{abstract}

\section{KEYWORDS}

Uncoded SER/BER estimation methods, Channel quality estimation, Link quality estimation over AWGN channel

\section{INTRODUCTION}

In order to fulfil the data services demand, the industry has to respond by designing more efficient technologies, planning for sophisticated networks, and maximizing the use of available resources. When the knowledge of wireless link quality/ channel conditions is available at the receiver, a variety of link quality based resource controls that achieve significant benefits in the total system throughput, system capacity and service quality can be deployed effectively.

Channel quality metrics specified in second and third generation standards include received signal strength, interference levels, and uncoded bit or symbol error rate. Precise estimation of uncoded BER or SER at the receiver-end can be used for effective power control, adaptive modulation, rate control, quality of service (QoS) and other resource management techniques. The primary requirements of uncoded SER or BER estimation are low measurement latency, high accuracy, and low computational complexity. However, the estimation accuracy and latency are a bit more important than the computational complexity as we can have the highly advanced processing units to handle the complexity.

This paper introduces a novel method for demodulated/ uncoded BER estimation using the accurate-bits sequence (ABS) of the new inner coding technique, proposed in [1]. Conventional uncoded SER estimation techniques use the test-pattern (TP) method and decode/reencode/compare (DRC) method [2] for the estimation. In TP method, a test pattern (pilot symbols) known to both the transmitter and receiver are sent over the wireless channel together with the information. The test pattern bits can either be continual or scattered over the frame. DOI : 10.5121/ijwmn.2015.7307 
However, the performance of TP method varies with the channel type and length of test pattern, and the performance of TP method serves as the upper bound on the DRC method. In contrast to TP method, the proposed estimation method uses the encoded information bits, which are not known to the receiver a priori, to estimate the demodulated BER. This paper explains the basic block diagram of simulation environment and also presents the simulation results of BER estimation over the AWGN channel.

\section{SER ESTIMATION METHODS}

In order to understand the difference between the proposed estimation method and conventional estimation method, it is important to take a short summary of a few conventionally used SER/BER estimation techniques. We will not discuss the mathematical detail of these estimation techniques as we are not using any part of it in the proposed estimation method. In [3] authors have analyzed the performance of a number of uncoded SER estimation techniques including a novel soft-information based SER estimation technique that was introduced in [4]. This section is mainly produced from [3] as its results are later used for the performance comparison. Uncoded SER/BER estimation methods can be categorized into two segments: Hard-information based methods and soft-information based methods.

\subsection{Hard-information Based Techniques}

\subsubsection{Test-Pattern (TP) Method}

In the TP method, the transmitter sends a test pattern that is known to the receiver. Upon reception of the test-pattern, the receiver performs a hard-decision MAP- demodulation on the received test symbols and records the number of discrepancies, or errors. Pictorial illustration of this method is shown in Figure 1. Although this method provides direct channel SER, it requires long test-patterns to predict the SER value with good accuracy. TP SER estimation is formulated as:

Estimation of $P_{s}=$ (Number of errors within test-pattern)/ Length of the test-pattern.

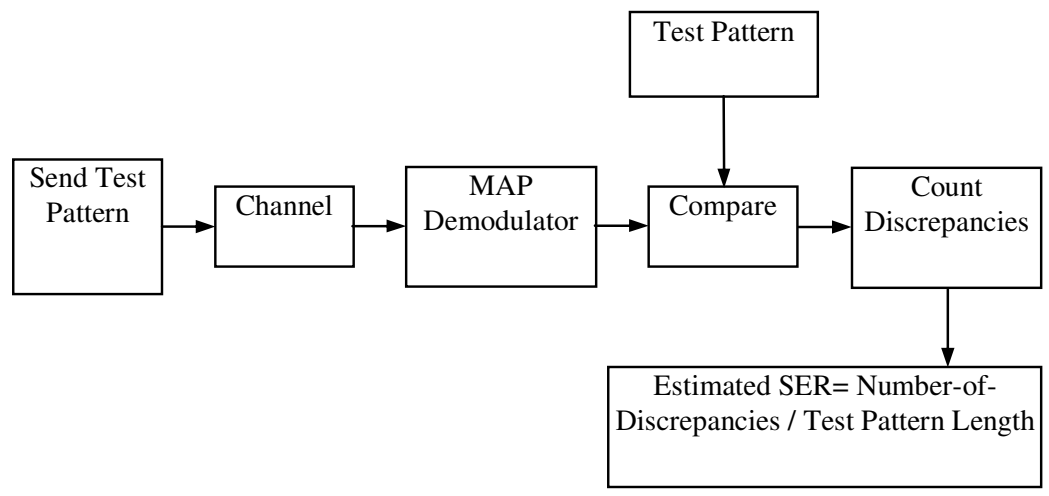

Figure 1. Pictorial illustration of the test-pattern SER estimation method

\subsubsection{Decode/Re-Encode/Compare (DRC) Method}

In the DRC method, the received symbols are first decoded (soft or hard, depending on the error correcting coding scheme). The decoded stream is re-encoded and compared to the MAP 
demodulated received symbol stream and the number of observed errors (discrepancies) is counted. The best performance the DRC method can achieve is that of the TP method since there are no post-decoding errors and the re-encoded stream is an exact replica of the transmitted stream.

\subsection{Soft-information Based Techniques}

\subsubsection{Direct SER Computation (DSC) Method}

In this method, the symbol error rate is calculated directly using a pre-computed SER formula, discussed in [2]. This technique assumes that the receiver has prior knowledge of the modulation scheme and the channel characteristics. However, the performance analysis of the DSC method is difficult to compute due to its dependency on modulation scheme and channel characteristics.

\subsubsection{Log-Likelihood (LLR) Method}

Log-likelihood ratio is usually used by soft decoders, such as Viterbi and Turbo decoders. The receiver can utilize this information, as proposed in [3], to estimate the SER. In LLR method, the received symbols are the actual encoded information symbols, which are not known to the receiver in advance.

\section{Proposed Estimation TeChNiQue}

An interesting and novel feature of the new channel coding technique, proposed in [1], is its bitwise reliability function (ABS). The original paper presented the experimental results for ABS using BPSK modulation scheme over the AWGN channel. A careful observation of simulation results, presented in [1], shows a linear relation between the percentage of valid bits in ABS and the simulated $E_{b} / N_{o}$ value (demodulated/ uncoded BER). In fact, this linear relation between demodulated BER and percentage of valid bits in ABS holds true for a wide range of simulated BER. Hence, the amount/percentage of valid bits in ABS (generated using forward path-1, forward path-2, reverse path- 1 and reverse path-2) can be used to estimate the demodulated BER over the AWGN channel. In order to exploit this linear relation, a lookup table is derived, which is known to the receiver in advance. Once the demodulated/uncoded BER estimate is found, it can be easily converted to appropriate uncoded SER value.

Table 1 shows the lookup table to estimate the demodulated BER using the percentage of valid bits in ABS. The lookup table is designed for the demodulated BER estimation range of 0.023 to 0.30. The relation between the percentage of valid bits in ABS and estimated BER is derived using a heuristic approach on the experimental data of ABS. Here, the ABS is generated using forward path-1, forward path-2, reverse path-1, and reverse path-2.

In order to get better estimation performance, it is essential to perform the estimation over small blocks and then take the overall average to find the final estimate for the entire block/frame. One can also use advanced mathematical tools to improve the estimation accuracy.

Table 1. Lookup table to estimate demodulated/uncoded BER

\begin{tabular}{|c|c|}
\hline $\begin{array}{c}\text { \% Of Valid } \\
\text { Bits in ABS }\end{array}$ & $\begin{array}{c}\text { Demodulated BER } \\
\text { estimate }\end{array}$ \\
\hline $53.00-53.92$ & 0.30 \\
\hline $53.92-54.29$ & 0.2873 \\
\hline
\end{tabular}


International Journal of Wireless \& Mobile Networks (IJWMN) Vol. 7, No. 3, June 2015

\begin{tabular}{|c|c|}
\hline $\begin{array}{c}\text { \% Of Valid } \\
\text { Bits in ABS }\end{array}$ & $\begin{array}{c}\text { Demodulated BER } \\
\text { estimate }\end{array}$ \\
\hline $54.29-54.94$ & 0.2762 \\
\hline $54.94-55.70$ & 0.2585 \\
\hline $55.70-56.69$ & 0.2459 \\
\hline $56.69-57.78$ & 0.2331 \\
\hline $57.78-58.91$ & 0.2202 \\
\hline $58.91-60.15$ & 0.2133 \\
\hline $60.15-61.64$ & 0.20 \\
\hline $61.64-63.33$ & 0.1860 \\
\hline $63.33-65.05$ & 0.1721 \\
\hline $65.05-66.95$ & 0.1595 \\
\hline $66.95-70.09$ & 0.14 \\
\hline $70.09-71.50$ & 0.125 \\
\hline $71.50-74.00$ & 0.105 \\
\hline $74.00-77.50$ & 0.09 \\
\hline $77.50-79.50$ & 0.079 \\
\hline $79.50-82.00$ & 0.066 \\
\hline $82.00-84.80$ & 0.056 \\
\hline $84.80-87.00$ & 0.0466 \\
\hline $87.00-89.20$ & 0.0373 \\
\hline $89.20-91.00$ & 0.0298 \\
\hline $91.00-92.70$ & 0.023 \\
\hline
\end{tabular}

\section{Simulation Results and Performance Comparison}

The shaded area in Figure 2 illustrates the simulation environment that is implemented in MATLAB. The new channel coding technique [1] is used as an inner code in serially concatenated configuration to transmit the information over the AWGN channel. At the receiver side hard-decision bits are used to generate the bit-wise reliability sequences (ABS). ABS and other decoded sequences are forwarded to the outer decoding block to retrieve the original information bits. The same ABS is used by the demodulated BER estimation block to estimate the channel quality. 


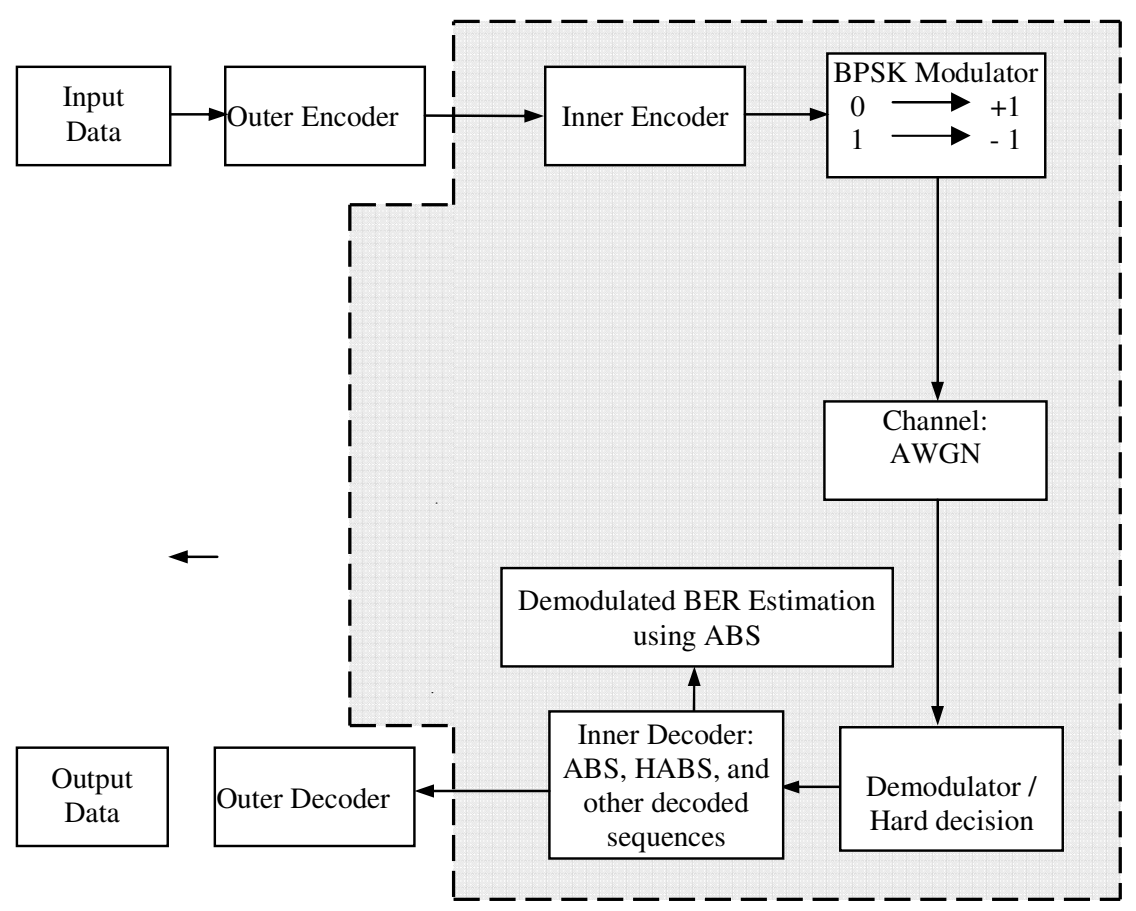

Figure 2. Block diagram of simulation environment

The estimation block divides the ABS into small size (100 bits) blocks and calculates the amount of the valid bits within each block. It then uses Table 1 to estimate the demodulated BER for each block. Finally an average is taken over all the blocks to find the final estimate of the demodulated BER over the entire block. It is apparent from the proposed estimation method that the overall computational complexity will be somewhat higher than that of the hard-information based estimation techniques (TP method).

Tables 2 and 3 show the symbol error rate (SER) estimation results, presented in [3], over the AWGN channel for TP method and LLR method respectively.

Table 2. Probability that the error in the SER estimation is within 10\% (TP Method, AWGN Channel, BPSK Modulation)

\begin{tabular}{|l|l|l|l|}
\hline $\begin{array}{l}\text { SNR } \\
(\mathbf{d B})\end{array}$ & \multicolumn{1}{|c|}{$\boldsymbol{P}_{\mathbf{s}}$} & $\boldsymbol{P}_{\mathbf{1 0} \%}(\boldsymbol{L}=\mathbf{1 0 0})$ & $\boldsymbol{P}_{\mathbf{1 0} \%}(\boldsymbol{L}=\mathbf{1 0 0 0})$ \\
\hline-4 & 0.26403 & 0.50311 & 0.94279 \\
\hline-2 & 0.2135 & 0.37309 & 0.89502 \\
\hline 0 & 0.15866 & 0.31692 & 0.83409 \\
\hline 2 & 0.10403 & 0.25488 & 0.72344 \\
\hline 4 & 0.0565 & 0.1638 & 0.58891 \\
\hline
\end{tabular}


Table 3. Probability that the error in the SER estimation is within 10\% (LLR Method, AWGN Channel, BPSK Modulation)

\begin{tabular}{|l|l|l|l|}
\hline $\begin{array}{l}\text { SNR } \\
(\mathbf{d B})\end{array}$ & \multicolumn{1}{|c|}{$\boldsymbol{P}_{\mathbf{s}}$} & $\boldsymbol{P}_{\mathbf{1 0} \%}(\boldsymbol{L}=\mathbf{1 0 0})$ & $\boldsymbol{P}_{\mathbf{1 0} \%}(\boldsymbol{L}=\mathbf{1 0 0 0})$ \\
\hline-4 & 0.26403 & 0.94951 & $\sim 1$ \\
\hline-2 & 0.2135 & 0.86029 & $\sim 1$ \\
\hline 0 & 0.15866 & 0.72549 & 0.99945 \\
\hline 2 & 0.10403 & 0.56641 & 0.98672 \\
\hline 4 & 0.0565 & 0.40306 & 0.90552 \\
\hline
\end{tabular}

Simulation results of the proposed estimation method over the AWGN channel are presented in Table 4. Estimation error mean is calculated by taking an average of absolute values of difference between the true error rate (simulated error rate) and the estimated error rate.

Estimation Error Mean= mean (abs (true error rate - estimated error rate))

This paper does not realize an end-to-end transmission solution for information bits; hence the simulations were performed for identical demodulated (uncoded) bit error probability over the AWGN channel. This makes it easier to compare the performance of the proposed estimation technique to that of the TP method and LLR method.

Table 4. Demodulated BER estimation performance (Frame size- 1200 bits, AWGN Channel, BPSK Modulation)

\begin{tabular}{|c|l|l|}
\hline $\begin{array}{c}\text { True } \\
\text { SER } \\
\boldsymbol{P}_{\boldsymbol{s}}\end{array}$ & $\begin{array}{c}\text { Estimation } \\
\text { Error Mean }\end{array}$ & $\begin{array}{c}\text { Required error tolerance in SER } \\
\text { estimation to guarantee } ~ \mathbf{9 9 \%} \\
\text { estimation accuracy }\end{array}$ \\
\hline 0.26403 & 0.0174 & $12.0 \%$ \\
\hline 0.21350 & 0.0131 & $11.0 \%$ \\
\hline 0.15866 & 0.0106 & $9.0 \%$ \\
\hline 0.10403 & 0.0083 & $6.0 \%$ \\
\hline 0.05650 & 0.0077 & $4.0 \%$ \\
\hline
\end{tabular}

It is clear from the simulation results that the proposed estimation method provides accurate estimate with low complexity at both high and low SNR values. The simulation results of the proposed estimation technique are better than the TP method and quite comparable to the LLR method in terms of estimation accuracy. Figure 3 shows the error estimation performance for 50 randomly transmitted frames over the AWGN channel. 


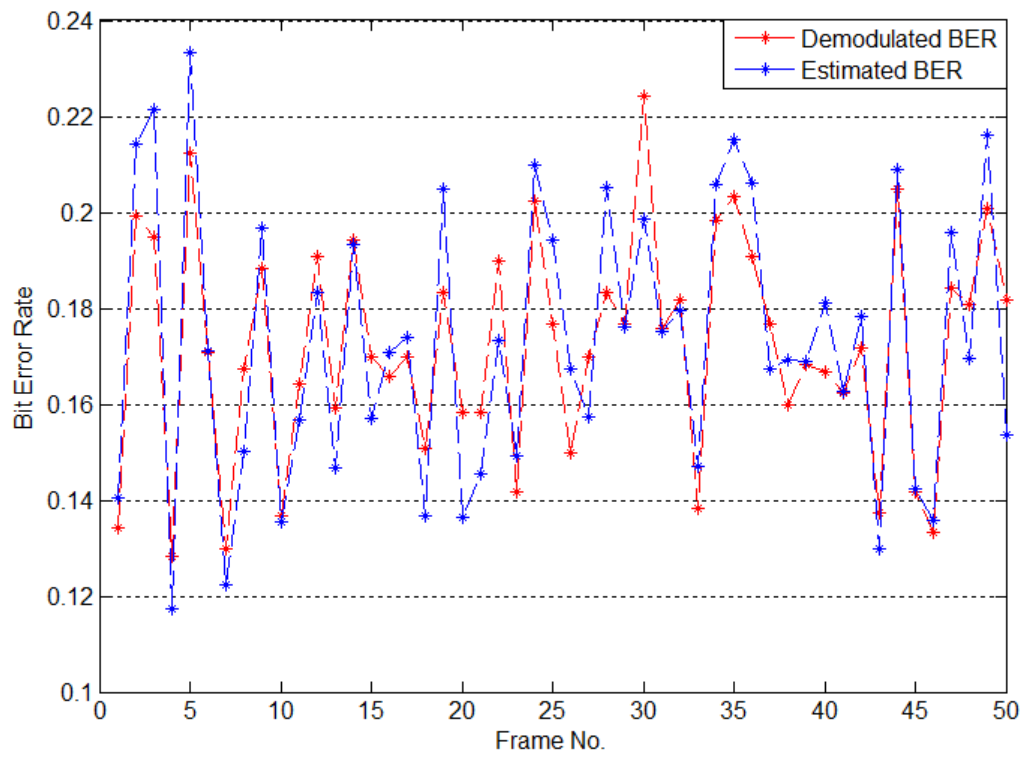

Figure 3. BER estimation performance over the AWGN channel

It is apparent form Figure 3 that the the estimated BER curve closely follows the demodulated (simulated) BER curve over the AWGN channel.

\section{CONCLUSION}

The paper presented a novel BER estimation method using the amount of valid bits in ABS that is generated during the decoding process of new channel coding technique [1]. The proposed estimation method provides a reliable estimate of demodulated/ uncoded BER for a wide range of simulated BER over the AWGN channel. One can also derive a lookup table for the ABS that is derived using a different combination of multiple decoded sequences. It is also apparent that the proposed estimation method is fairly simple as we only need to calculate the amount of valid bits in ABS to provide the estimate. As the lookup table for demodulated BER estimation is derived using a heuristic approach, one can always use curve fitting techniques to further improve the estimation accuracy.

\section{REFERENCES}

[1] M. Patel and A. Annamalai, (Feb. 2015) "A new channel coding technique to approach the channel capacity," International Journal of Wireless \& Mobile Networks (IJWMN), Vol. 7, No. 1, pp. 23-42.

[2] S. P. Emeott, (1993) "Method for determining a channel quality metric in a receiver," http://uspto.gov, U.S. patent 5493584.

[3] W. K. M. Ahmed and K. Balachandran, (Nov. 2005) "Uncoded Symbol Error Rate estimation: methods and analysis," IEEE Transaction on Vehicular Technology, vol. 54, no. 6.

[4] K. Balachandran and S. Nanda, (Mar. 2006) "Bit Error Probability Estimation in a Communicaion System Using Soft Decision Information at the Receiver,” U.S. patent 7020185. 


\section{Authors}

Mahesh Patel received the B.S. degree in Electronics and Telecommunication Engineering from North Maharashtra University, India, and M.S. degree in Electrical Engineering from University of Texas at Arlington, United States of America in 2004 and 2007, respectively. $\mathrm{He}$ is currently working towards his $\mathrm{PhD}$. degree in the Department of Electrical and Computer Engineering at the Prairie View A\&M University, a member of Texas A\&M

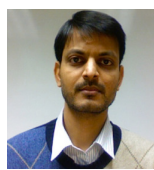
University System. His current research interests include low rate channel codes, forward error correction codes and error estimation codes.

Dr. Annamalai is presently the Director of Center of Excellence for Communication Systems Technology Research, a Texas A\&M Board of Regents approved University Research Center at the Prairie View A\&M University, and a tenured professor in the Department of Electrical and Computer Engineering. He has over 16 years of research/teaching experience in wireless communications at Motorola, University of Victoria, Air Force Research Laboratory, Virginia Tech and PVAMU with over 200 peer-reviewed publications and 6 book chapters. Dr. Annamalai has been honored by his colleagues on numerous occasions for his excellence in research including winning the 2011 Roy G. Perry College of Engineering Outstanding Faculty (Research) Award, IEEE Leon Kirchmayer Prize Paper award, ASEE/AFOSR Summer Faculty Fellowships, NSERC Doctoral Prize, CAGS/UMI Distinguished Doctoral Dissertation Award, IEEE VTS/Motorola Daniel E. Noble Fellowship, among others. He had served on the Editorial Boards of four IEEE journals/transactions in the last 12 years, and has helped to organize a few major IEEE conferences on wireless communications including serving in the capacity of Technical Program Chair of the 2002 IEEE Vehicular Technology Conference in Vancouver, Canada. His current research interests include cooperative spectrum sensing, compressive sensing, cross-layer design for scalable multimedia transmission and cooperative wireless communications. 